\title{
Synthesis and Evaluation of New Nitrone-Based Benzoxazinic Antioxidants ${ }^{+}$
}

\author{
Stefania Marano ${ }^{1}$, Cristina Minnelli ${ }^{2}$, Giovanna Mobbili ${ }^{2}$, Emiliano Laudadio ${ }^{1}$ \\ and Pierluigi Stipa ${ }^{1, *}$ \\ 1 Dipartimento S.I.M.A.U., Università Politecnica delle Marche, via Brecce Bianche, 60131 Ancona, Italy; \\ s.marano@staff.univpm.it (S.M.); e.laudadio@staff.univpm.it (E.L.) \\ 2 Dipartimento di Scienze della Vita e dell'Ambiente (DISVA), Università Politecnica delle Marche, \\ via Brecce Bianche, 60131 Ancona, Italy; c.minnelli@staff.univpm.it (C.M.); g.mobbili@staff.univpm.it \\ (G.M.) \\ * Correspondence: p.stipa@staff.univpm.it; Tel.: +39-071-2204409 \\ + Presented at the 1st International e-Conference on Antioxidants in Health and Disease, 01-15 December \\ 2020; Available online: https://cahd2020.sciforum.net/.
}

Published: 30 November 2020

\begin{abstract}
Oxidative stress is often the cause of a wide range of human chronic pathologies including but not limited to stroke, cardiovascular and neurodegenerative diseases. In this setting, increasing efforts are currently being made to the design and synthesis of new derivatives with enhanced antioxidant efficacy. Among all the potential molecules of interest, synthetic nitrone spin-traps have attracted a great deal of research attention, particularly due to their dual function: as effective inhibitors of oxidative stress and damage, and as analytical tool for the detection and characterization of free radicals by means of Electron Paramagnetic Resonance (EPR) spectroscopy Spin Trapping Technique. In this study, two derivatives of benzoxazinic nitrones (3-aryl-2Hbenzo[1,4]oxazin-N-oxides) bearing an electron-withdrawing methyl-acetate group on the benzo moiety (in para and meta positions respect to the nitronyl function) were synthesized. Their in vitro antioxidant activity was evaluated by means of the $\alpha, \alpha$-diphenyl- $\beta$-picrylhydrazyl radical (DPPH) scavenging assay, and their inhibitory effects on the erythrocyte hemolysis induced by the water soluble free radical initiator 2,2'-azobis(2-amidinopropane) dihydrochloride (AAPH) compared. In addition, EPR was employed to monitor the decay profiles of DPPH to evaluate the kinetic behavior of the different antioxidants tested. Results showed that the presence and the position of the electron-withdrawing methyl-acetate group strongly affects the radical scavenging activity of nitrones. In particular, the newly synthesized para-substituted derivative when compared to both the meta-substituted isomer and the unsubstituted parent compound, acts as more effective antioxidant both in cell and cell-free systems. Overall, these results clearly show that the introduction of an electron-withdrawing group on the phenyl moiety significantly increased the antioxidant capacity of benzoxazinic nitrones, thus showing exciting opportunities in the search of new therapeutic agents in the treatment of diseases associated with oxidative stress.
\end{abstract}

Keywords: nitrone; DPPH; antioxidant; electron paramagnetic resonance

\section{Introduction}

The ability of nitrones to act as good radical traps has been widely researched over the last decades [1-5]. For years, nitrones have been used as efficient analytical tool for the detection and characterization of free radicals using EPR Spin Trapping Technique, based on their ability to quickly 
The 1st International Electronic Conference on Antioxidants in Health and Disease, 1-15 December 2020 trap short-lived free radicals and afford persistent and identifiable aminoxyl spin adducts [6-9]. Due to this unique ability, nitrones have attracted considerable attention as potential therapeutic agents for the treatment of many pathologies where reactive oxygen species (ROS) are implicated, such as cardiovascular diseases, cancer, aging, cerebral ischemia and various neurodegenerative diseases [10-12]. Although it would be reasonable to attribute their biological action to their spin trapping activity, a clear picture of the actual mechanism of action of nitrones has not yet been reported. On the contrary, a large number of investigations has pointed out that more than one mechanisms may be involved and the free radical trapping activity may be not so likely to be their primary action [10,13-15]. As part of our ongoing research on the reactivity of benzoxazinic nitrones, a series of 3aryl-2H-benzo[1,4]oxazin-N-oxides have been previously synthesized [16], and their reactivity towards several carbon- and oxygen-centered radicals widely explored [3,10]. Results revealed that they act as efficient spin traps for a wide range of free radicals and their activity has been demonstrated to be higher than the commercially available N-tert-Butyl- $\alpha$-phenylnitrone (PBN) [10]. Since, over the past years, several para-substituted nitrones $[15,17,18]$, bearing electron-withdrawing groups have shown improved radical detection and antioxidant properties, in this study we synthesized two constitutional isomers of benzoxazinic nitrones bearing a methyl-acetate group on the benzo moiety (in para- and meta-position) with the aim to enhance the reactivity of the already studied unsubstituted parent compound. Moreover, this study aims to get additional insights into their antioxidant mechanism of action.

\section{Materials and Methods}

\subsection{Materials and Characterization}

$\alpha$-diphenyl-picrylhydrazyl (DPPH) and 2,2-azobis (2-amidinopropane hydrochloride) (AAPH) were obtained from Merck Co. (Stenheim, Germany). All the other reagents and chemicals were of analytical grade for biochemical purposes or HPLC grade. All the solutions were prepared in ultrapure MilliQ water to prevent metal contamination. Electrospray ionization mass spectra were recorded on a Finnigan Navigator LC/MS single-quadrupole mass spectrometer in $\mathrm{EI}^{+}$mode, cone voltage $25 \mathrm{~V}$ and capillary voltage $3.5 \mathrm{kV}$, by injecting samples previously dissolved in methanol. The ${ }^{1} \mathrm{H}$ and ${ }^{13} \mathrm{C}$ NMR spectra were recorded on a Varian Gemini 400 spectrometer in $\mathrm{CDCl}_{3}$ at 400 and 100 $\mathrm{MHz}$, respectively. Chemical shifts are reported in ppm relative to residual solvent signals $(\delta=7.24$ and $77.30 \mathrm{ppm}$ for ${ }^{1} \mathrm{H}$ and ${ }^{13} \mathrm{C}$ NMR, respectively), and coupling constants (J) in Hz. FT-IR spectra were collected on a PerkinElmer Spectrum GX FT-IR spectrophotometer equipped with ATR single reflection diamond. Measurements were performed with a resolution of $2 \mathrm{~cm}^{-1}$, 16 scans over 4000 $500 \mathrm{~cm}^{-1}$ range. Samples were directly placed on the measuring surface without requiring any preparation. Background adsorption spectrum was recorded each time for correction. Spectra were analyzed on Spectrum 5.3.1 (Perkin-Elmer) operating software. Isotropic X-band EPR spectra were recorded on a Bruker EMX/Xenon spectrometer system equipped with a microwave frequency counter and an NMR Gauss meter for field calibration; for g-factor determination, the whole system was standardized with a sample of perylene radical cation in concentrated sulfuric acid ( $\mathrm{g}$-factor $=$ 2.00258).

\subsection{Synthetic Procedure}

The two constitutional isomers of benzoxazinic nitrones (3-aryl-2H-benzo[1,4]oxazin-N-oxides) (2-3) bearing a methyl-acetate group on the benzo moiety and the unsubstituted derivative (1) were prepared with a slight modification of the previously reported two-step method [16,19], as illustrated in Scheme 1: alkaline condensation between the appropriate 2-nitrophenol and the 2bromoacetophenone, followed by reductive cyclization $(\mathrm{Zn} / \mathrm{NH} 4 \mathrm{Cl})$.

6-(methoxycarbonyl)-3-phenyl-2H-benzo[b][1,4]oxazine 4-oxide (2): ${ }^{1} \mathrm{H} \mathrm{NMR}\left(400 \mathrm{MHz}, \mathrm{CDCl}_{3}\right) \delta(\mathrm{ppm})$ : 3.91 (s, 3H, 6-COOCH 3$), 5.38$ (s, 2H, 2-CH2), 7.03 (d, J = 8.49 Hz, 1H, C8), 7.24 (CDCl $), 7.48$ (d, J = 7.15 
The 1st International Electronic Conference on Antioxidants in Health and Disease, 1-15 December 2020

$\mathrm{Hz}, 1 \mathrm{H}, 8-\mathrm{CH}), 8.03$ (d, J = 8.47 Hz, 5H, 3-CH), 8.22 (d, J = 8.06 Hz, 2H, 5-7-CH). ${ }^{13} \mathrm{C}$ NMR (100 MHz, $\left.\mathrm{CDCl}_{3}\right) \delta$ (ppm): 66.56, 76.77, 76.99, $77.31\left(\mathrm{CDCl}_{3}\right), 115.99,121.04,122.64,128.06,128.51,130.68,131.17$, 149.55. Mp: 118-120. IR (KBr, cm $\left.{ }^{-1}\right): 1714(v(\mathrm{C}=\mathrm{O})$ ester $), 1435(\delta(\mathrm{CH} 3)$ methoxy), $1618(v(\mathrm{C}=\mathrm{N})), 1266$ $\left(v(\mathrm{~N}-\mathrm{O}) \mathrm{N}\right.$-oxide). ESI-MS $(\mathrm{m} / \mathrm{z})$ of $\mathrm{C}_{16} \mathrm{H}_{13} \mathrm{NO}_{4}: \mathrm{Mr}=283.278$, found 283.322.

7-(methoxycarbonyl)-3-phenyl-2H-benzo[b][1,4]oxazine 4-oxide (3): ${ }^{1} \mathrm{H} \mathrm{NMR}\left(400 \mathrm{MHz}, \mathrm{CDCl}_{3}\right) \delta(\mathrm{ppm})$ : $3.92\left(\mathrm{~s}, 3 \mathrm{H}, 7-\mathrm{COOCH}_{3}\right), 5.35\left(\mathrm{~s}, 2 \mathrm{H}, 2-\mathrm{CH}_{2}\right), 7.24\left(\mathrm{CDCl}_{3}\right), 7.48(\mathrm{~m}, \mathrm{~J}=7.35 \mathrm{~Hz}, 1 \mathrm{H}, 8-\mathrm{CH}), 7.65-7.81(\mathrm{~m}$, $\mathrm{J}=8.47 \mathrm{~Hz}, 5 \mathrm{H}, 3-\mathrm{CH}), 8.23$ (d, J = 8.40 Hz, 2H, 6-5-CH). ${ }^{13} \mathrm{C} \mathrm{NMR}\left(100 \mathrm{MHz}, \mathrm{CDCl}_{3}\right) \delta$ (ppm): 66.67, 76.67, 76.98, $77.30\left(\mathrm{CDCl}_{3}\right), 117.44,121.17,123.79,128.13,131.19,149.27 . \mathrm{Mp}: 124-125 . \mathrm{IR}\left(\mathrm{KBr}, \mathrm{cm}^{-1}\right)$ : $1713(v(\mathrm{C}=\mathrm{O})$ ester $), 1437(\delta(\mathrm{CH} 3)$ methoxy), $1608 v(\mathrm{C}=\mathrm{N}), 1265(v(\mathrm{~N}-\mathrm{O}) \mathrm{N}-\mathrm{oxide})$. ESI-MS $(\mathrm{m} / z)$ of $\mathrm{C}_{16} \mathrm{H}_{13} \mathrm{NO}_{4}:\left[\mathrm{Mr}+\mathrm{H}^{+}\right]=284.29$, found 284.57 .

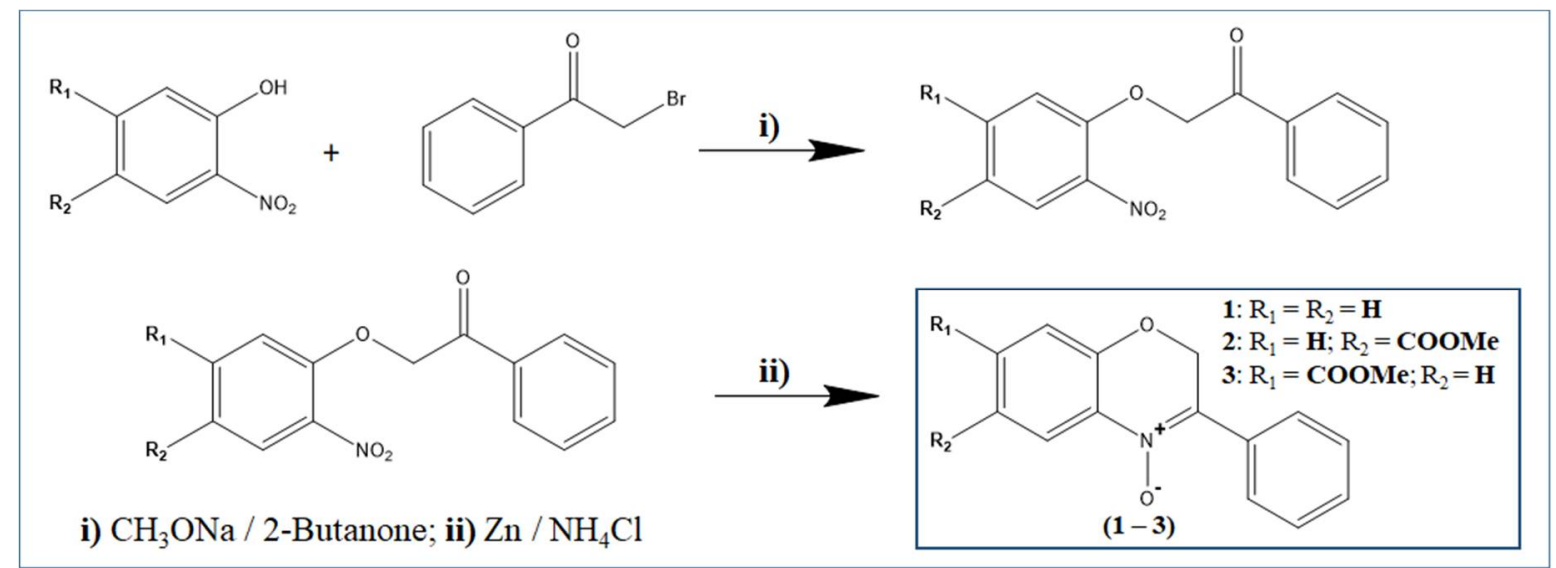

Scheme 1. Synthesis of the target benzoxazinic nitrones (1-3).

\subsection{DPPH Spectrophotometric Radical Scavenging Assay}

The free radical scavenging activity of nitrones was determined by their ability to reduce the free DPPH radical as previously described [20], with minor modifications. For the spectrophotometric evaluations, antioxidant and DPPH solutions in methanol were mixed (antioxidant $50 \mu \mathrm{M}, \mathrm{DPPH} 100$ $\mu \mathrm{M}, \mathrm{FC}$ ), shaken vigorously and incubated for $30 \mathrm{~min}$ and $60 \mathrm{~min}$ in the dark. The absorbance at 517 nm was determined with a spectrophotometer (BioTek Synergy HT MicroPlate Reader Spectrophotometer) against a blank which lacked in DPPH.

The percent inhibition of the DPPH radical by antioxidants was calculated according to the following Equation (1):

$$
\text { Inhibition ratio }(\%)=\left[\left(\mathrm{A}_{\text {control }}-\mathrm{A}_{\text {sample }}\right) / \mathrm{A}_{\text {control }}\right] \times 100
$$

where $\mathrm{A}_{\text {control }}$ is the absorbance of the control obtained by adding into the DPPH methanol solution a methanol aliquot equal to the antioxidant solution volume. Asample is the absorbance of the reaction solution at $30 \mathrm{~min}$. Each sample was measured in triplicate. Mean and standard deviation $(n=3)$ were calculated.

\subsection{DPPH Signal Quenching by EPR}

The antioxidant ability of nitrones was evaluated using DPPH quenching approach by EPR spectroscopy. All the experiments were carried out in the dark to avoid photochemical effects on the otherwise stable DPPH radical. EPR spectra were recorded on a Bruker EMX spectrometer system operating at $100 \mathrm{KHz}$, equipped with a NMR microwave frequency counter. The incubation mixtures contained DPPH at constant concentration of $800 \mu \mathrm{M}$ and nitrones in two different nitrone:DPPH molar ratios (1:2 and 1:4) in a total volume of $200 \mu \mathrm{L}$ benzenic solution. The kinetic profiles were obtained following the decay in the EPR signal intensity as a function of time (7200 s) using the 1D_TimeSweep spectra collection mode. The decay in the DPPH spectra intensity occurs the e EPR- 
The 1st International Electronic Conference on Antioxidants in Health and Disease, 1-15 December 2020

silent diamagnetic counterpart is formed; the test is therefore representative of the capacity of nitrones to react with stable organic radicals. The amplitude of the first line of DPPH spectra was taken for registration of kinetics. Measurements were run at least in triplicate.

\subsection{Determination of Erythrocyte Hemolysis Induced by AAPH}

Erythrocyte oxidative hemolysis induced by AAPH and its inhibition in the presence of nitrones was performed as previously described [21]. $5 \mathrm{~mL}$ of human blood in EDTA was washed three times in PBS (pH 7.4) by centrifugation at $1000 \mathrm{~g}$ for 10 minutes. The resulting pellet was resuspended with 6-fold PBS (pH 7.4). 0.5 mL of erythrocyte suspension was added to $0.25 \mathrm{~mL}$ of PBS nitrone solutions obtaining a final antioxidant concentration of $0.012,0.5$ and $0.2 \mathrm{mM}$. The mixtures were incubated for $1 \mathrm{~h}$ at $37^{\circ} \mathrm{C}$ and then, $0.25 \mathrm{~mL}$ of PBS was added with or without AAPH (final concentration AAPH, $50 \mathrm{mM}$ ) followed by incubation for $12 \mathrm{~h}$ at $37^{\circ} \mathrm{C}$ in a gently shaking incubator.

$100 \%$ lysis was determined by adding $0.15 \mathrm{~mL}$ of a $1 \%$ Triton X-100 solution while only PBS was added in the negative control. After sedimentation of the unlysed erythrocytes by centrifugation $\left(1000 \mathrm{~g}, 15 \mathrm{~min}, 4^{\circ} \mathrm{C}\right)$, the supernatant was transferred to a microtiter plate and diluted 1:4. The hemoglobin absorption was determined at $540 \mathrm{~nm}$ using the Synergy HT microplate reader spectrophotometer (BioTek, Winooski, VT, USA). The percent hemolysis was determined using the following Equation (2):

$$
\% \text { Hemolysis }=\left[\left(\mathrm{A}-\mathrm{A}_{0}\right) /\left(\mathrm{A}_{\text {total }}-\mathrm{A}_{0}\right)\right] \times 100
$$

where $\mathrm{A}$ is the absorbance of the test well, $\mathrm{A}_{0}$ is the absorbance of the negative control, and Atotal is the absorbance of the positive control. The mean values of three replicates were reported.

\subsection{DFT Calculations}

Density Functional Theory (DFT) calculations were carried out using the GAUSSIAN 09 suite of programs [22], taking advantage of the resources available at Cineca Supercomputing Center [23]. All calculations on paramagnetic species were carried out with the unrestricted formalism, giving $S^{2}$ $=0.7501 \pm 0.0003$ for spin contamination (after annihilation). Thermodynamic quantities were computed at $298 \mathrm{~K}$ by means of frequency calculations performed employing the M06-2X functional in conjunction with the $6-31+\mathrm{G}(\mathrm{d}, \mathrm{p})$ basis set, starting from molecular geometries computed at the B3-LYP/6-31G(d) level of theory. In Frequency calculations, no negative values (imaginary frequencies) have been found, demonstrating that all quantities were referred to geometry minima. All calculations are referred to the gas phase.

\subsection{Statistical Analysis}

Data are presented as mean \pm S.D. Statistical comparison of differences between antioxidants tested was carried out using Student's $t$-test. Values of $p<0.05$ were considered statistically significant.

\section{Results and Discussion}

\subsection{Synthesis and Structural Characterization}

The unsubstituted nitrone, 3-phenyl-2H-benzo[1,4] oxazine 4-oxide, is a known compound, thus both the details of its synthesis and a full structural characterization can be found in the literature $[16,19]$. In this study two derivatives of this compound bearing an electron-withdrawing methylacetate group on the benzo moiety were synthetized and their structure confirmed by mass spectrometry (MS), infrared spectroscopy (IR) and nuclear magnetic resonance spectroscopy $\left({ }^{1} \mathrm{H}-\right.$ NMR and ${ }^{13}$ C-NMR) (see Section 2.2). 


\subsection{DPPH Scavenging Activity of Nitrones}

Nitrones are known spin traps that are capable of trapping free radicals (carbon-, oxygen- and nitrogen-centered radicals) via addition reactions at the $\mathrm{C}=\mathrm{N}$ double bond of the nitronyl function, thus, encouraging their use as pharmacological agents against oxidative stress-mediated pathologies. However, the mechanism by which nitrones exert their antioxidant activity is still not completely clear. Besides the well-documented radical trapping ability of nitrones, a large body of investigations suggest that more than one mechanism may be involved [10,13-15]. In the specific case of DPPH, Scheme 2 summarizes all the possible reaction mechanisms that could take place between DPPH and the nitrones under investigation. In a typical DPPH assay, nitrones are therefore expected to quench DPPH free radicals via a spin trapping mechanism, giving rise to a nitroxide spin adduct (pathway (a)). However, quenching of DPPH radical by either hydrogen atom transfer (HAT) and/or sequential electron transfer (SET) are also possible. The H-donating ability could be associated to both the formation of the corresponding hydroxylamine tautomer and the potentially removable hydrogens in position 2, which could be abstracted by DPPH radicals, followed by potential formation of spin adducts by further reaction between another DPPH radical and the corresponding radicals formed (pathway (b)). Moreover, potential monoelectronic redox behavior (SET) of either the resulting nitroxide or oxoammonium compound should be taken in consideration (pathways (a-b)). The relative reactivity of nitrones towards DPPH radicals was evaluated by both EPR and spectrophotometry.

a)

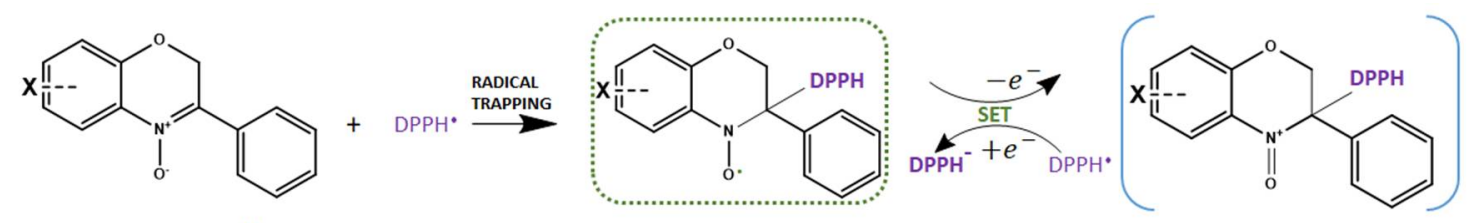

b)
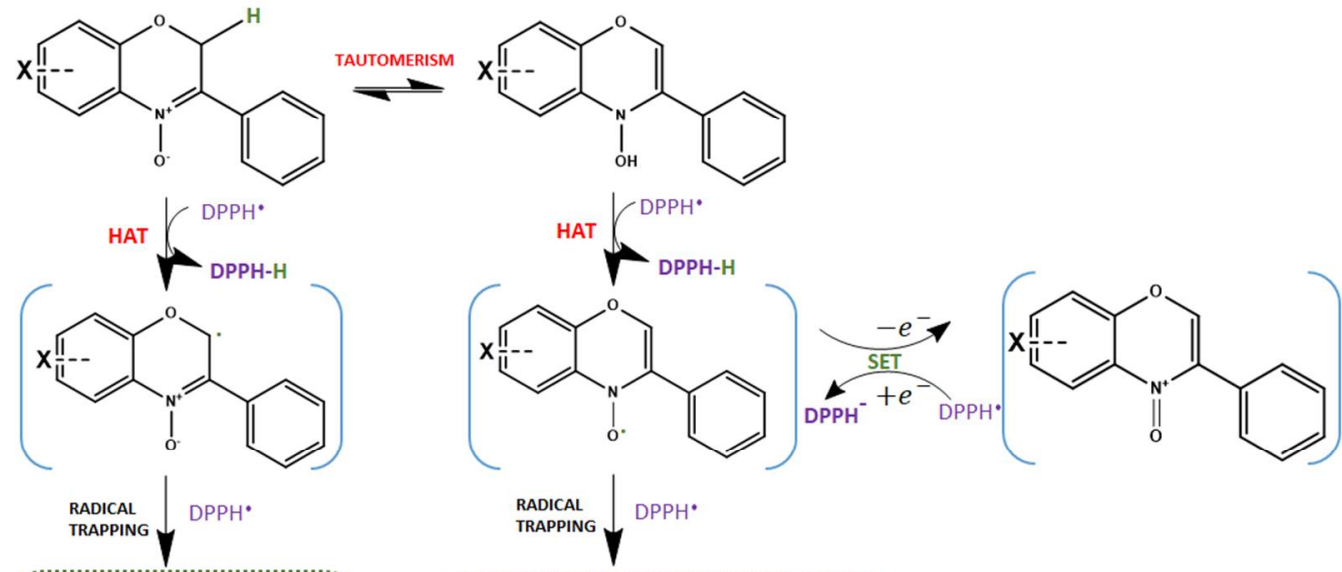<smiles></smiles>

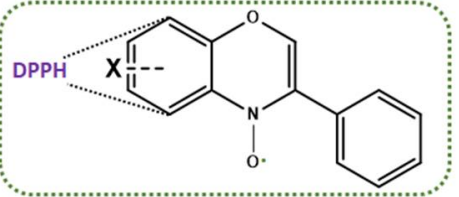

Scheme 2. An overview of the potential mechanisms involving nitrones and DPPH; (a) formation of nitroxide spin adduct at $\alpha$-carbon atom and subsequent SET (Single Electron Transfer); (b) HAT (Hydrogen Atom Transfer) pathways with formation of spin adducts in position 2 and at the benzoring and SET pathway from hydroxylamine-oxoammonium products.

\subsubsection{DFT Calculations}

The computed values collected in Table 1 indicates that all the benzoxazinic nitrones under investigation show comparable reactivity (i.e., similar spin trapping ability) toward all the radicals 
The 1st International Electronic Conference on Antioxidants in Health and Disease, 1-15 December 2020

tested, showing easier reaction with C-radicals compared to peroxyl or alkoxyl radicals. This analysis has been already reported for the unsubstituted parent compound [10]. However, differences could be found in the IP values (in gas phase) between the two isomers ( 13 Kcal smaller for the metasubstituted), indicating that a SET pathway may potentially be easier for the meta-substituted compound compared to the para-substituted one.

Table 1. Computed Gibbs free energies changes $(\Delta G)$ of radical addition reactions to nitrones and Ionization potential (IP); all quantities are reported in $\mathrm{Kcal} / \mathrm{mol}$ and referred to the gas phase.

\begin{tabular}{|c|c|c|c|c|c|c|}
\hline Nitrone & \multicolumn{5}{|c|}{ Radical $(\Delta G)$} & IP \\
\hline & Me & OMe & OOMe & $\mathrm{OH}$ & $\mathrm{OOH}$ & \\
\hline 1 & -42.51 & -38.69 & -16.30 & -54.34 & -25.06 & 174.19 \\
\hline 2 & -42.59 & -36.91 & -19.29 & -52.79 & -24.66 & 163.28 \\
\hline 3 & -43.10 & -39.72 & -19.27 & -54.60 & -24.88 & 176.41 \\
\hline
\end{tabular}

\subsubsection{DPPH Quenching by EPR and UV-Assay}

Figure 1A-C shows the EPR kinetic profiles for the reaction between nitrones and DPPH in the two different molar ratios (1:2 and 1:4). As can be clearly seen from the corresponding traces, paraand meta-substituted nitrones (A-B) are able to quench DPPH and the extent of the signal reduction is dose-dependent, achieving up to approximately $37 \%$ and $15 \%$ decrease in the DPPH activity at the highest concentrations (1:2 nitrone:DPPH molar ratio), respectively. On the other hand, the unsubstituted nitrone 1 shows no activity toward DPPH and its addition led to an initial clear growth of the DPPH signal amplitude, up to $\sim 5 \%$ for the 1:2 molar ratio, suggesting a pro-oxidant behavior taking place at the beginning $(C)$. Regarding derivatives 2 and 3 , the presence and the position of the electron-withdrawing methyl-acetate group appears to strongly affect their activity. In particular, derivative 3 , bearing the substituent in para position with respect to the nitronyl function, exhibited higher anti-DPPH radical activity than the meta-substituted isomer.

In order to see whether the DPPH scavenging activity behaviors of nitrones were reproducible and independent by potential solvent-solute interactions, the decay profiles in the absorbance of the reaction mixtures at 1:2 nitrone:DPPH molar ratio were also measured at $517 \mathrm{~nm}$ in a polar solvent (methanol). Figure 1D shows the percentage inhibition of DPPH based on the measured absorbance values taken at two different incubation times (at 30 and $60 \mathrm{~min}$ ). It is clear that both the decay trend of the DPPH radical for nitrones 2 and 3 and the signal growth for 1 observed by UV/VIS spectroscopy are in good agreement with those observed by EPR in benzene. This indicates a good reproducibility of the result from the two methods employed, despite of the very different polarity of the solvents used (methanol and benzene).

In order to get additional insights into the potential mechanisms underlying the DPPH scavenging activity of nitrones, the reaction mixtures were analyzed by liquid chromatography-mass spectrometry (LC-MS). Results indicated that only for nitrone 3 there were clear evidences of spin adduct formations with DPPH (ions at $m / z 701\left[M_{7-\text { Coome }}+M_{D P P H}+N a\right]$ and $985\left[2 M_{7-\text { coome }}+\right.$ $\left.M_{D P P H}+N a\right]$,) while no additional peaks were found in the mass spectra of the other two nitroneDPPH reaction mixtures. As mentioned before, potential spin adducts could arise from either direct trapping of the free radical at its $\alpha$-carbon atom of the $\mathrm{N}$-oxide $\left(\mathrm{C}=\mathrm{N}^{+}-\mathrm{O}^{-}\right)$group, giving rise to a paramagnetic nitroxide adduct (pathway (a)) or by hydrogen atom transfer (HAT) and subsequent formation of spin adducts in position 2 and/or at the phenyl ring (pathway (b)). Since no additional paramagnetic species were detected during the EPR experiment, pathway (a) may be excluded as nitroxide adducts are typically very stable and persistent, implying that further insights should be searched within pathway (b) for the para-substituted nitrone. The lower activity of nitrone 2 compared to nitrone 3, could be linked to its higher tendency to be oxidized (see Section 3.2.1): in this case, since the nitrone could be partially oxidized, its amount available for other possible reaction with DPPH could be less than expected. 
A)

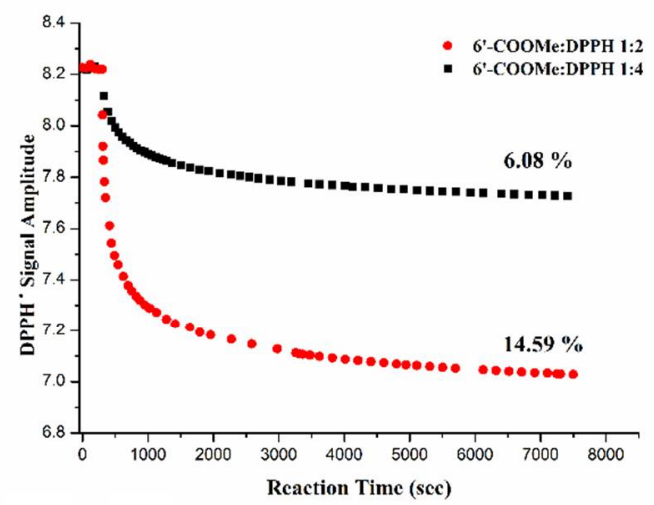

C)

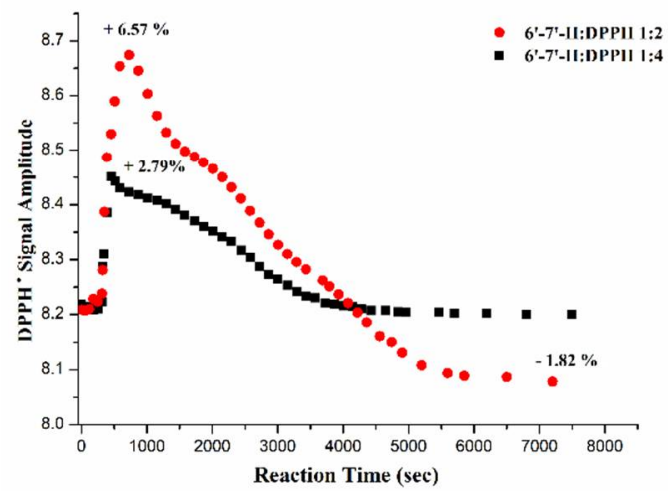

B)

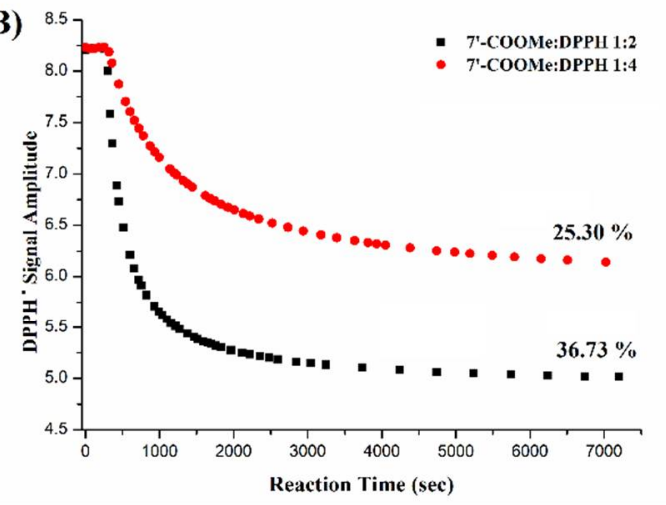

D)

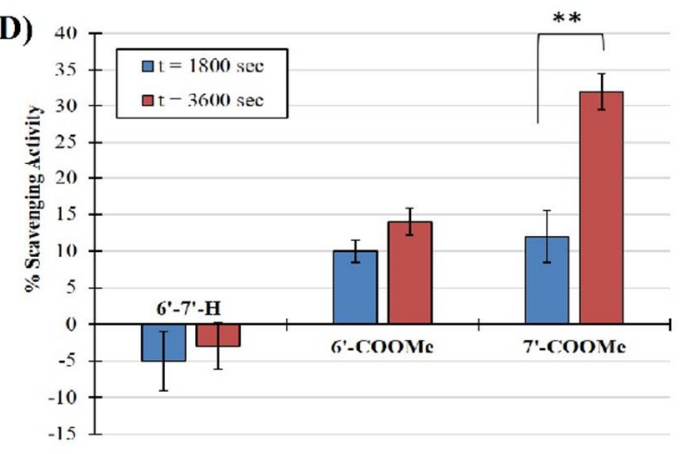

Figure 1. (A-C) Kinetic profiles for the reaction of 200 and $400 \mu \mathrm{M}$ nitrone solutions with $800 \mu \mathrm{M}$ DPPH in benzene at $23^{\circ} \mathrm{C}$; (D) Spectrophotometric evaluation of free radical scavenging activity of nitrones by DPPH assay. Results are expressed as the mean \pm standard deviation $(n=5) .{ }^{* *} p<0.001$.

\subsection{Protection against AAPH-Induced Hemolysis}

The hemolysis of erythrocytes has been extensively used as an ex vivo model in the study of oxidative stress-induced alteration of cell membranes. AAPH is a water-soluble radical initiator, which generates, after thermal decomposition, a constant flux of peroxyl radicals, responsible for inducing lipid peroxidation and oxidation of membrane proteins. This leads to the formation of hemolytic holes in the membrane. Erythrocytes were therefore pre-treated with antioxidant compounds followed by addition of AAPH. As shown in Figure 2, the percentage of survival erythrocyte population was related to the concentration of the antioxidant tested with the parasubstituted nitrone having the highest ability to protect the erythrocyte cells from AAPH-induced hemolysis, confirming what observed in the previous paragraphs. 


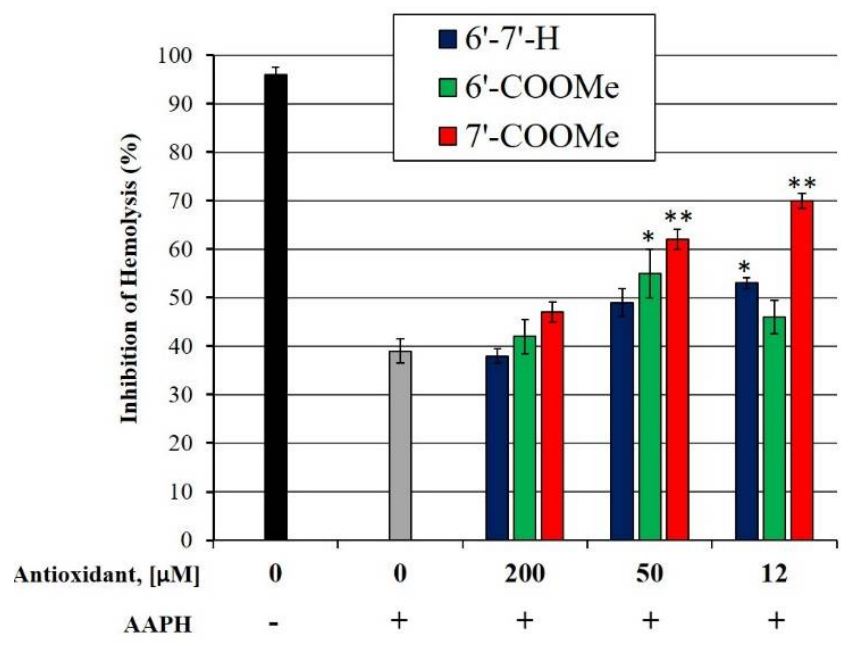

Figure 2. Hemolysis of human erythrocytes induced by AAPH. RBC suspensions were treated with nitrones followed incubation with $50 \mathrm{mM}$ AAPH at $37{ }^{\circ} \mathrm{C}$. ${ }^{*} p<0.05$ and ${ }^{* *} p<0.001$ with respect untreated RBCs.

\section{Conclusions}

In this study, two new derivatives of benzoxazinic nitrone family were synthesized and the effects of electron withdrawing substituents on the benzo-ring on their antioxidant activity were explored in vitro. More in details, the reactivity of the two derivatives bearing a methyl-acetate group in benzoxazine 6 and 7 positions were evaluated using both typical DPPH scavenging assay and oxidative hemolysis inhibition test with human erythrocytes. Results demonstrated that in both cases, the presence and the position of such a substituent appears to affect the nitrones antioxidant activity. In particular, the derivative substituted in para position with respect to the nitronyl function, exhibited higher anti-DPPH radical potency and higher $\%$ of hemolysis inhibition than both the metasubstituted isomer and the unsubstituted parent compound. Relative to the DPPH activity, MS data of the reaction mixtures showed evidences of spin adducts formation only for the para-substituted compound, which may at least account for its higher activity against DPPH radicals. On the other hand, even if less active, the meta-substituted isomer showed a lower (computed) ionization potential, indicating a greater tendency to be oxidized and reduce DPPH radicals via a SET mechanism. Although the antioxidant activity trend for all compounds are in fair accordance in both assays, further investigations and analyses are now in progress and will be reported in due course. In this regard, its worth pointing out that care should be taken while estimating the antioxidant activity of newly synthesized compounds by means of DPPH assay, whose radical nature could interfere with the antioxidants under investigation.

Funding: This research was funded by Università Politecnica delle Marche.

Conflicts of Interest: The authors declare no conflict of interest.

\section{References}

1. Harbour, J.R.; Issler, S.L.; Hair, M.L. Singlet Oxygen and Spin Trapping with Nitrones. J. Am. Chem. Soc. 1980, 102, 7778-7779.

2. Alberti, A.; Carloni, P.; Eberson, L.; Greci, L.; Stipa, P. New insights into N-tert-butyl- $\alpha$-phenylnitrone $(\mathrm{PBN})+$ as a spin trap. Part 2.1 the reactivity of PBN and 5,5-dimethyl-4,5-dihydropyrrole N-oxide (DMPO) toward N-heteroaromatic bases. J. Chem. Soc. Perkin Trans. 2 1997, 887-892, doi:10.1039/a608004i.

3. Stipa, P. OH radical trapping with benzoxazine nitrones: A combined computational and spectroscopic study. Tetrahedron 2013, 69, 4591-4596, doi:10.1016/j.tet.2013.04.021. 
The 1st International Electronic Conference on Antioxidants in Health and Disease, 1-15 December 2020

4. Gruber, N.; Piehl, L.L.; De Celis, E.R.; Díaz, J.E.; García, M.B.; Stipa, P.; Orelli, L.R. Amidinoquinoxaline Noxides as novel spin traps. RSC Adv. 2015, 5, 2724-2731, doi:10.1039/c4ra14335c.

5. Ivanov, A.V.; Martynovskaya, S.V.; Shcherbakova, V.S.; Ushakov, I.A.; Borodina, T.N.; Bobkov, A.S.; Vitkovskaya, N.M. Ambient access to a new family of pyrrole-fused pyrazine nitrones via 2-carbonyl- N allenylpyrroles. Org. Chem. Front. 2020, doi:10.1039/d0qo00762e.

6. Janzen, E.G.; Blackburn, B.J. Detection and Identification of Short-Lived Free Radicals by an Electron Spin Resonance Trapping Technique. J. Am. Chem. Soc. 1968, 90, 5909-5910.

7. Dembkowski, L.; Finet, J.P.; Frjaville, C.; Le Moigne, F.; Maurin, R.; Mercier, A.; Pages, P.; Stipa, P.; Tordo, P. B-Phosphorylated Five Membered Ring Nitroxides. Synthesis and Epr Study. Free Radic. Res. Commun. 1993, 58, 4468, doi:10.3109/10715769309056s23.

8. Beyer, M.; Fritscher, J.; Feresin, E.; Schiemann, O. Synthesis of novel aromatic nitroxides as potential DNA intercalators. An EPR spectroscopical and DFT computational study. J. Org. Chem. 2003, 68, 2209-2215, doi:10.1021/jo026742n.

9. Stipa, P.; Cipolletti, R. DFT calculations as a powerful tool for ESR spin trapping experiments. Magn. Reson. Chem. 2017, 55, 559-562, doi:10.1002/mrc.4549.

10. Astolfi, P.; Carloni, P.; Marini, M.G.; Mobbili, G.; Pisani, M.; Stipa, P. Benzoxazinic nitrones and nitroxides as possible antioxidants in biological systems. RSC Adv. 2013, 3, 22023-22030, doi:10.1039/c3ra44073g.

11. Ferraz, M.; Mano, R.; Oliveira, D.; Maia, D.; Silva, W.; Savegnago, L.; Lenardão, E.; Jacob, R. Synthesis, Antimicrobial, and Antioxidant Activities of Chalcogen-Containing Nitrone Derivatives from (R)citronellal. Medicines 2017, 4, 39, doi:10.3390/medicines4020039.

12. Marco-Contelles, J. Recent Advances on Nitrones Design for Stroke Treatment. J. Med. Chem. 2020, doi:10.1021/acs.jmedchem.0c00976.

13. Chakraborty, K.B.; Scott, G.; Yaghmour, H. Mechanisms of antioxidant action: The antioxidant activity of nitrones in polypropylene. J. Appl. Polym. Sci. 1985, 30, 3267-3281, doi:10.1002/app.1985.070300811.

14. Chioua, M.; Sucunza, D.; Soriano, E.; Hadjipavlou-Litina, D.; Alcázar, A.; Ayuso, I.; Oset-Gasque, M.J.; González, M.P.; Monjas, L.; Rodríguez-Franco, M.I.; et al. $\alpha$-aryl-N-alkyl nitrones, as potential agents for stroke treatment: Synthesis, theoretical calculations, antioxidant, anti-inflammatory, neuroprotective, and brain-blood barrier permeability properties. J. Med. Chem. 2012, 55, 153-168, doi:10.1021/jm201105a.

15. Deletraz, A.; Zéamari, K.; Hua, K.; Combes, M.; Villamena, F.A.; Tuccio, B.; Callizot, N.; Durand, G. Substituted $\alpha$-Phenyl and $\alpha$-Naphthlyl- N- tert-butyl Nitrones: Synthesis, Spin-Trapping, and Neuroprotection Evaluation. J. Org. Chem. 2020, 85, 6073-6085, doi:10.1021/acs.joc.0c00563.

16. Battistoni, P.; Bruni, P.; Fava, G. 3-phenyl-2H-1,4-benzoxazine-4-oxides-I. Synthesis and reduction. Tetrahedron 1979, 35, 1771-1775, doi:10.1016/0040-4020(79)88007-2.

17. Rosselin, M.; Tuccio, B.; Pério, P.; Villamena, F.A.; Fabre, P.L.; Durand, G.; Fabre, P.L. Electrochemical and Spin-Trapping Properties of para-substituted $\alpha$-Phenyl-N-tert-butyl Nitrones. Electrochim. Acta 2016, 193, 231-239, doi:10.1016/j.electacta.2016.02.038.

18. Deletraz, A.; Zéamari, K.; Di Meo, F.; Fabre, P.L.; Reybier, K.; Trouillas, P.; Tuccio, B.; Durand, G. Reactivities of MeO-substituted PBN-type nitrones. New J. Chem. 2019, 43, 15754-15762, doi:10.1039/c9nj03805a.

19. Astolfi, P.; Marini, M.; Stipa, P. Radical trapping properties of 3-aryl-2H-benzo[1,4]oxazin-4-oxides. J. Org. Chem. 2007, 71, 8677-8682, doi:10.1021/jo071212i.

20. Minnelli, C.; Laudadio, E.; Galeazzi, R.; Rusciano, D.; Armeni, T.; Stipa, P.; Cantarini, M.; Mobbili, G. Synthesis, characterization and antioxidant properties of a new lipophilic derivative of edaravone. Antioxidants 2019, doi:10.3390/antiox8080258.

21. Liu, H.; Ma, J.; Wu, H. Detoxifying effects of ultrafiltration fractions of Dendrobium aphyllum peptides on chemical and AAPH-induced oxidative stress. RSC Adv. 2017, 7, 48913-48924, doi:10.1039/c7ra08957k.

22. Frisch, M.J.; Trucks, G.W.; Schlegel, H.B.; Scuseria, G.E.; Robb, M.A.; Cheeseman, J.R.; Scalmani, G.; Barone, V.; Mennucci, B.; Petersson, G.A.; et al. Gaussian09 Revision D.01; Gaussian 09 Revis. C.01; Gaussian Inc.: Wallingford, CT, USA, 2010. 
The 1st International Electronic Conference on Antioxidants in Health and Disease, 1-15 December 2020

23. Cineca Supercomputing Center. via Magnanelli 6/3, I-40033 Casalecchio di Reno, Bologna, Italy. Available online: http://www.cineca.it/ HPSystems (accessed on).

Publisher's Note: MDPI stays neutral with regard to jurisdictional claims in published maps and institutional affiliations.

(C) 2020 by the authors. Submitted for possible open access publication under the terms and conditions of the Creative Commons Attribution (CC BY) license (http://creativecommons.org/licenses/by/4.0/). 